ESTRATÉGIA 


\section{A PRODUÇÃO EM INTERNACIONALIZAÇÃO DO ENSINO SUPERIOR: UMA ANÁLISE A PARTIR DA BASE DA WEB OF SCIENCE}

THE PRODUCTION IN INTERNATIONALIZATION OF HIGHER EDUCATION: AN ANALYSIS FROM THE BASIS OF WEB OF SCIENCE

Fernando Gazzoni

Universidade Federal de Santa Maria

Flavia Luciane Scherer

Universidade Federal de Santa Maria

Natália Pavanelo Pivetta

Universidade Federal de Santa Maria

Maíra Nunes Piveta

Universidade Federal de Santa Maria
Andréa Bach Rizzatti

Universidade Federal de Santa Maria
Data de submissão: 28 jan. 20| 8. Data de aprovação: I5 jun. 2018. Sistema de avaliação: Double blind review. Universidade FUMEC / FACE. Prof. Dr. Henrique Cordeiro Martins. Prof. Dr. Cid Gonçalves Filho.

\section{RESUMO}

A internacionalização do ensino superior pode ser analisada sobre diversos prismas, diante disso a presente pesquisa justifica-se à medida que busca identificar e analisar os principais aspectos das publicações no campo de estudo, apontando os hot topics da temática. Para a realização deste estudo, utilizou-se a pesquisa bibliométrica na base de dados da Web of Science, compreendendo as publicações sobre o tema no período de 2007 a 2016 . Foram encontradas I I46 publicações, que evidenciaram a existência de um grande número de autores e instituições que publicam sobre a temática, bem como diversas fontes de publicação. Nota-se ainda que grande parte dos estudos estão voltados para a área da educação e possuem o inglês como idioma predominante. Os termos "globalização" e "universidade" foram identificados como hot topics no estudo da temática, possivelmente por serem considerados termos correlatos ao objeto de estudo e não uma área específica de estudo.

\section{PALAVRAS-CHAVE}

Internacionalização do Ensino Superior. Globalização. Hot topics. Web of Science. Bibliometria. 


\section{ABSTRACT}

The higher education internationalization can be analyzed on several aspects, therefore the present research is justified as it seeks to identify and analyze the main publications aspects in this field of study, pointing out the hot topics of the subject. In order to carry out this study, bibliometric research was used in the Web of Science database, comprising publications on the subject from 2007 to 20I6.There were II 46 publications, which evidenced the existence of a large number of authors and institutions that publish on the subject, as well as several sources of publication. Furthermore, most of the studies are focused on education and have English as the predominant language. The terms "globalization" and "university" were identified as hot topics in the theme of study, possibly because they were considered terms correlated to the object of study and not a specific area of study

\section{KEYWORDS}

Internationalization of higher education. Globalization. Hot topics. Web of science. Bibliometry.

\section{INTRODUÇÃO}

O mundo de hoje está experimentando uma intensificação sem precedentes da interconexão econômica, cultural, política e social (JACKSON, 2008). Esse fenômeno, denominado globalização, possui impacto que "se estende muito além do domínio dos negócios e empresa, também afeta o tecido cultural das sociedades e instituições educacionais" (JACKSON, 2008). Assim, como espaço gerador de conhecimento, as instituições de ensino superior têm contribuído para esse processo, visto que têm "aproximado os povos e têm feito com que as populações tenham acesso muito rápido e direto ao que está ocorrendo nos lugares mais longínquos do globo, gerando um acelerado processo de internacionalização" (STALLIVIERI, 2002).

Frente a esse cenário de globalização atual, a internacionalização do ensino é de suma importância para a inovação disruptiva da educação (DUTRA; MARANHÃO,
2017). Deste modo, "a internacionalização das instituições de ensino superior, através das diferentes formas de cooperação, tem sido o gatilho para a melhoria da qualidade do ensino e da pesquisa" (STALLIVIERI, 2002). Por meio da mobilidade de estudantes, professores e de gestores é possível com que se intensifiquem os laços transnacionais, estabelecendo conexões e criando redes de saber universal (STALLIVIERI, 2002), com isso, promove-se o avanço científico de todas as nações (CAPES, 20I0).

Assim, de acordo com Jackson (2008), a internacionalização do ensino superior pode assumir diversas formas, como o intercâmbio estudantil entre faculdades, estudo e moradia no exterior, estágios ou aprendizagem de serviços em um país estrangeiro, currículos "globalizados" sobre campus de origem, educação em línguas estrangeiras, área ou estudos culturais, programas de educação internacional ou comparativa e campus no exterior. Ainda 
segundo o autor, devido a essas várias opções de internacionalização de ensino um número cada vez maior de universidades está incentivando seus alunos a participar de programas de estudos no exterior, seja por uma oportunidade de crescimento (JACKSON, 2008). Ou ainda, por uma questão de sobrevivência, para competir em níveis de igualdade com as melhores instituições de ensino superior nacionais e estrangeiros (STALLIVIERI, 2002).

Neste sentido, para que haja essa mobilidade acadêmica, a temática de internacionalização do ensino superior implica a mobilização de diversos agentes, entre eles: estado, instituições de ensino nacionais e internacionais, gestores educacionais e comunidade acadêmica (DUTRA; MARA$\mathrm{NHÃO}, 2017)$. Além desta mobilização dos diversos agentes é necessário que os países tenham políticas de mobilidade, programas de financiamentos, metas para internacionalização, critérios de avaliação (DUTRA; MARANHÃO, 2017), entre outros métodos para que haja uma atitude favorável ao desdobramento de seu processo e êxito na execução, garantindo assim, o fortalecimento da competitividade nos mercados mundiais (STALLIVIERI, 2002).

A internacionalização das instituições de ensino superior existe há muito tempo. Segundo Stallivieri (2002) "o caráter internacional das universidades está presente desde a Idade Média com a criação das primeiras escolas europeias”. Porém, como "as atividades internacionais das universidades se expandiram dramaticamente em volume, escopo e complexidade nas últimas duas décadas" (ALTBACH; KNIGHT, 2007), muitos pesquisadores vêm se interessando por esse campo de estudo (DE WIT, 20I I ;ALTBACH; KNIGHT, 2007;
KNIGHT, 2008; STALLIVIERI, 2002).

Em virtude do caráter multifacetado do tema, relacionado às diversas motivações existentes pelo campo de pesquisa, resulta uma ampla gama de trabalhos que buscam estudar uma realidade específica, no entanto esta situação resulta em uma pequena quantidade de autores que conhecem o tema com profundidade (LIMA; CONTEL, 20II). Em vista disso, pelo crescente número de estudos sobre a internacionalização de ensino superior e por existir uma ampla gama de possibilidades neste campo, a presente pesquisa justifica-se à medida que busca identificar e analisar os principais aspectos das publicações sobre internacionalização do ensino superior. Para a realização deste estudo, foram pesquisadas publicações referentes ao período de 2007 a 2016 na base de dados Web of Science. Por meio dessa análise, será possível ampliar o conhecimento sobre essa temática e, então, compreender o estado-da-arte deste campo de estudo.

Para isso, o presente artigo encontra-se estruturado em cinco seções, sendo esta introdução a primeira delas. $\mathrm{Na}$ segunda seção são abordados os principais conceitos acerca da temática internacionalização do ensino superior a fim de facilitar a compreensão e desenvolvimento da pesquisa. Em seguida, são apresentados os procedimentos metodológicos utilizados no trabalho. Na quarta seção são demonstradas as análises e discussões dos resultados. Por fim, na última seção são expostas as considerações finais, e em sequência as referências bibliográficas utilizadas neste estudo.

\section{REFERENCIALTEÓRICO}

As atividades internacionais das instituições de ensino superior se expandiram 
drasticamente em volume, escopo e complexidade nas duas últimas décadas (ALTBACH; KNIGHT, 2007). Neste sentido, a internacionalização é um termo que está sendo usado cada vez mais para discutir a dimensão internacional das universidades e, mais amplamente, a educação na pósgraduação (KNIGHT, 2008, p. I).

Sendo assim, existe um discurso e debate há mais de 20 anos sobre a definição da internacionalização do ensino. Esse é um termo que significa coisas diferentes para pessoas diferentes, portanto existe uma confusão sobre seu significado. Para algumas pessoas significa uma série de atividades internacionais (mobilidade acadêmica, vínculos internacionais e novos programas acadêmicos). Para outras significa a entrega de educação para outros países. Para muitos significa a inclusão de uma dimensão internacional no currículo e processo de aprendizagem. Outros veem a internacionalização como comércio no ensino superior. E ainda, existe uma confusão frequente sobre a relação entre internacionalização e globalização (KNIGHT, 2008, p. I).

Neste sentido, de acordo com De Wit (20II) existem diversos autores que se dedicam a escrever sobre a complexa relação entre globalização e internacionalização no ensino superior, dentre eles Ulrich Teichler (2004), Peter Scott (2005), Philip Altbach (2006), Jane Knight (2008), Felix Maringe, Nick Foskett (20I0) e outros. Deste modo, julga-se importante diferenciar os termos globalização e internacionalização do ensino superior. Para Knight (2008, p. x), "a globalização é o processo que está aumentando o fluxo de pessoas, cultura, ideias, valores, conhecimento, tecnologia e economia através das fronteiras", podendo ter consequências positivas ou negativas para diferentes nações. Já a internacionalização do ensino superior "é o processo de integração da dimensão internacional, intercultural e global entre o propósito, funções (ensino, pesquisa e serviço) e entrega do ensino superior nos níveis institucional e nacional" (KNIGHT, 2008, p. xi).

Corroborando com Knight (2008), Dutra e Maranhão (20I7) dissertam que "o termo internacionalização pode ser encontrado em diversas áreas e possui diferentes conceitos". Para Morosini, Fernandes, Leite et al. (2016) a internacionalização está no cerne do ente universitário e é fator de legitimação da circulação do conhecimento e da formação de recursos humanos. Para Marrara (2007) "a internacionalização envolve um fluxo de pessoas, informações e, eventualmente, recursos que se movem, tanto na direção das instituições estrangeiras com as quais se mantêm laços de cooperação acadêmica, quanto na direção oposta".

Diversos são os estudos que analisam a internacionalização sob a ótica das instituições de ensino superior.A autora Stallivieri (2002), por exemplo, realizou um estudo sobre o processo de internacionalização nas instituições de ensino superior. Deste estudo, a autora identificou alguns entraves que podem atrasar o processo de internacionalização, tais como:

A ausência da sensibilização da comunidade acadêmica, atitudes passivas por parte do corpo docente e discente da instituição, inexistência de estratégias claras para a internacionalização, a falta de reconhecimento cultural, indisponibilidade ou inviabilidade de adaptação das comunidades estrangeiras, barreiras linguísticas ou elevado distan- 
ciamento geoeducacional, entre outros (STALLIVIERI, 2002).

Assim, esses são alguns dos fatores que podem comprometer o sucesso na realização de bons programas de cooperação, sejam eles assumidos bilateralmente ou financiados pelos órgãos governamentais. $A$ autora ainda salienta que cada universidade possui autonomia para definir suas estratégias de acordo com suas necessidades e objetivos de sua instituição, porém, para o estabelecimento dessas estratégias de internacionalização, as instituições devem observar alguns pontos que podem delimitar a sua área de atuação (STALLIVIERI, 2002).

Marrara (2007), em seu estudo, identificou alguns dos objetivos da internacionalização sendo eles a aquisição de renome internacional em benefício da instituição de ensino superior e políticas de internacionalização como ferramentas a serviço da formação de docentes, pesquisadores e discentes. $O$ autor também explicou que apesar de não haver uma fixação juridicamente vinculante dos objetivos que devam caracterizar o processo de internacionalização acadêmica no Brasil, existem parâmetros constitucionais, legais e ministeriais que tratam da avaliação da educação superior, sendo a Capes (Coordenação de Aperfeiçoamento de Pessoal de Nível Superior), agência federal de fomento à pesquisa, um dos órgãos responsáveis pela avaliação.

Além disso, o autor classificou a internacionalização das instituições de ensino superior em duas formas. Na primeira forma, internacionalização passiva, "predominam o envio de discentes, docentes e pesquisadores para instituições estrangeiras, bem como a publicação dos trabalhos científicos desses autores em periódicos internacionais, externos à instituições de ensino superior brasileira", os quais buscam capacitação em instituições estrangeiras. Já a segunda forma, internacionalização ativa, "se caracteriza pelo recebimento de docentes, pesquisadores e discentes estrangeiros e pela participação desses agentes em cursos e periódicos da instituição de ensino superior nacional", isso depende do engajamento e da abertura das instituições nacionais (MARRARA, 2007).

Os autores Altbach e Knight (2007) realizaram um estudo cujo o objetivo foi compreender os estímulos mais importantes para a internacionalização acadêmica.A partir desse estudo, os autores identificaram alguns dos incentivos para internacionalização, tais como: o lucro (alguma espécie de ganho, seja em questões monetárias ou desejo de aumento de pesquisa e conhecimento); provisão de acesso e absorção da demanda (atender a proporções de jovens que desejam estudar em outros locais); internacionalização tradicional (universidades que já participam de atividades tradicionais há muito tempo); internacionalismo europeu (programas da UE em que os estudantes são encorajados a estudar no exterior dentro da UE); internacionalização dos países em desenvolvimento (procuram atrair estudantes estrangeiros para suas universidades para melhorar a qualidade e a composição cultural do corpo estudantil, ganham prestígio e ganham renda) e; internacionalização individual (estudantes que são autofinanciados).

Os autores Borges e Amal (2016) realizaram um levantamento sobre as diversas maneiras em que pode ocorrer o processo de internacionalização das instituições de ensino superior. Deste estudo, os autores verificaram que existem 10 maneiras para uma instituição de ensino superior se internacionalizar, conforme exposto no quadro I. 
QUADRO 1 - Formas de internacionalização

\begin{tabular}{|c|c|}
\hline Formas de Internacionalização & Autor(es) \\
\hline Intercâmbio de alunos & $\begin{array}{c}\text { Kerr (1990), Paige (2003), Altbach e King (2007), Huang (2007), Murphy (2007), } \\
\text { Bégin-Caouette (2012), Olatokun e Utulu (2012), Baernholdt (2013). }\end{array}$ \\
\hline Intercâmbio de professores & $\begin{array}{c}\text { Kerr (1990), Paige (2003), Altbach e King (2007), Murphy (2007), } \\
\text { Bégin-Caouette (2012). }\end{array}$ \\
\hline Pesquisa em conjunto & Huang (2007), Coryell et al. (2012). \\
\hline Publicação e revistas estrangeiras & Bogotch e Maslin-Ostrowski, (2010), Byun e Kim (2011). \\
\hline Currículos adaptados & $\begin{array}{c}\text { Kerr (1990), Paige (2003), Huang (2007), Bogotch e Maslin- } \\
\text { Ostrowski, (2010), Coryell et al. (2012). }\end{array}$ \\
\hline Programas adaptados & Altbach e King (2007). \\
\hline Cursos a distância & Paige (2003), Altbach e King (2007). \\
\hline Presença física (unidades no exterior) & Paige (2003). \\
\hline Visita de professores & Bégin-Caouette (2012), Coryell et al. (2012). \\
\hline Alunos estrangeiros & \\
\hline
\end{tabular}

Fonte: Borges; Amal (2016)

Deste modo, conforme apresentado no quadro I, das 10 maneiras que uma instituição de ensino superior pode se internacionalizar, o intercâmbio de alunos e professores são os assuntos mais explorados pelos pesquisadores.

Além deste levantamento, que foi uma das partes do estudo, os autores Borges e Amal (2016) tinham como objetivo principal verificar se a distância psíquica é levada em consideração no processo de internacionalização de cursos stricto sensu (cursos de mestrado e doutorado) das instituições de ensino superior brasileiras. Desse estudo, os autores constataram que a escolha do país ocorre pela rede de contato dos professores, sendo que a distância psíquica não é decisiva para a escolha de novos mercados e a falta de conhecimento sobre o mercado exterior não é um obstáculo. Ainda, os autores constataram nesse estudo que os principais determinantes para a internacionalização são: "reconhecimento (social e internacional), credibilidade, busca pela excelência, visibilidade do curso e ampliação do conhecimento" (BORGES; AMAL, 2016).
Dado o exposto, a internacionalização do ensino superior está cada vez mais presente na literatura, motivo este que estimulou o presente estudo cujo o objetivo foi de identificar e analisar os principais temas estudados (hot topics) sobre a internacionalização do ensino superior entre o período de 2007 a 2016.

\section{MÉTODO}

O presente estudo desenvolveu-se sobre uma perspectiva da pesquisa bibliométrica, com cunho predominantemente quantitativo. Optou-se por este método objetivando ampliar o conhecimento referente às principais publicações da área e compreender os principais aspectos relacionados ao estudo da internacionalização do ensino superior. De acordo com Araújo (2006), a bibliometria consiste numa técnica quantitativa e estatística de medição dos índices de produção e disseminação de conhecimento científico vislumbrando a análise quantitativa da informação bibliográfica. Para Macedo, Casa Nova e Almeida (2009) ela ajuda a conhecer o estágio em que uma pesquisa em determinada área se encontra. 
A análise dos dados quantitativos compreendeu uma análise estatística descritiva que buscou investigar as características gerais das publicações, tais como: total de publicações, autores, áreas temáticas, tipos de documentos, título das fontes, ano das publicações, instituições, agências de financiamento, idiomas e países.

Para definição dos hot topics utiliza-se o índice m, calculado através do índice $h-b$. Banks (2006) contribuiu com o índice $h-b$, uma extensão do $h$-index. $O h$-index (índice -h) foi proposto por Hirsch (2005), em sua pesquisa denominada An index to quantify an individual's scientific research output, como forma de caracterizar a produção científica de um pesquisador. Já o índice $h$-b, por sua vez, é obtido através do número de citações de um tópico ou combinação em determinado período, listados em ordem decrescente de citações. Ele é encontrado em publicações que tenham obtido um número de citações igual ou maior à sua posição no ranking. Também explica o cálculo do índice $\mathrm{m}$, o qual é obtido através da divisão do índice $h$-b pelo período de anos que se deseja obter informações (n).

O quadro 2 apresenta a classificação do índice $\mathrm{m}$ e a relação com os hot topics de acordo com a classificação proposta por Banks (2006).

\section{Coleta de dados e etapas da pesquisa}

Inicialmente foram definidos os termos principais para pesquisa e a base de dados onde seria realizada. Optou-se por utilizar a base de dados da Web of Science, em virtude do seu extenso banco de dados, possuindo atualmente cerca de 12.000 periódicos indexados (CAPES 2017). Referente aos termos de busca, optou-se por replicar os termos "internacionalização" e "ensino superior", no entanto utilizou-se os termos em inglês, uma vez que a pesquisa dos termos em língua portuguesa não apresentou nenhum resultado. Em análise inicial verificou-se a existência de duas formas de grafia para a palavra internacionalização, "internationalization" e "internationalisation", variando de acordo com a região onde foi desenvolvido o estudo. Desta maneira utilizou-se o símbolo "\$” para realização da pesquisa, uma vez que a utilização desse símbolo permite abranger ambos resultados.

A presente pesquisa dividiu-se em quatro etapas. A primeira etapa consistiu em pesquisar como tópicos os termos "internationali\$ation" e "higher education" na base de dados da Web of Science, definindo-se o período de 10 anos, a escolha deste período de análise pauta-se em estudos desenvolvidos que utilizaram o mesmo período de tempo para identificação de comportamento dos estudos de determinada área, como os estudos desenvolvidos por DalSoto, Alves e Souza (20 I6), Figueiró e Raufflet (20I5) e Raasch et al. (20I3). Desta

\section{QUADRO 2 - Definições para a classificação dos hot topics}

\begin{tabular}{|c|l|}
\hline Índice $\mathrm{m}$ & \multicolumn{1}{c|}{ Tópico/Combinação } \\
\hline $0<\mathrm{m} \leq 0,5$ & $\begin{array}{l}\text { Pode ser de interesse para pesquisadores em um campo específico de pesquisa, o qual engloba uma comu- } \\
\text { nidade pequena; }\end{array}$ \\
\hline $0,5<\mathrm{m} \leq 2$ & $\begin{array}{l}\text { Provavelmente pode se tornar um "hot topic" como área de pesquisa, no qual a comunidade é muito grande ou } \\
\text { o tópico/combinação apresenta características muito interessantes; }\end{array}$ \\
\hline $\mathrm{m}>2$ & $\begin{array}{l}\text { É considerado um "hot topic", tópico exclusivo com alcance não apenas na sua própria área de pesquisa e é } \\
\text { provável que tenha efeitos de aplicação ou características únicas. }\end{array}$ \\
\hline
\end{tabular}

Fonte: Banks (2006) 
maneira o período selecionado para o desenvolvimento desta análise compreende o intervalo de 2007 até 2016. Ressalta-se que na seleção da base de dados foi escoIhida somente a principal coleção da Web of Science. Realizada a pesquisa inicial, foram identificadas as características gerais das publicações, tais como: ano das publicações, autores, países, instituições, idiomas, áreas de pesquisa e fontes de publicação.

$\mathrm{Na}$ segunda etapa, após uma breve leitura dos trabalhos encontrados, foram identificados os principais tópicos relacionados ao tema, dentre os tópicos identificados, foram selecionados os 20 principais para realização do cruzamento com os tópicos "internationali\$ation" e "higher education". Para seleção dos tópicos utilizou-se como principal critério a relação com o termo pesquisado.

$\mathrm{Na}$ terceira etapa foi realizado o cruzamento previsto na etapa anterior, os critérios de pesquisa foram os mesmos utilizados na primeira etapa, pesquisa por tópicos, pelo período de 10 anos (20072016) e somente na base principal da Web of Science.

$\mathrm{Na}$ quarta, e última etapa, com base nos resultados da etapa anterior, foram calculados os índices $\mathrm{h}$-b e m, o que possibilitaram a identificação dos hot topics referente a internacionalização do ensino superior.

A figura I busca ilustrar as etapas propostas no estudo.

\section{ANÁLISE E DISCUSSÃO DOS RESULTADOS}

Foram encontradas II46 publicações relacionadas ao tema internacionalização do ensino superior na base de dados da Web of Science, no período compreendido entre 2007 e 2016, com base nas publicações encontradas serão apresentadas as características gerais das publicações, bem

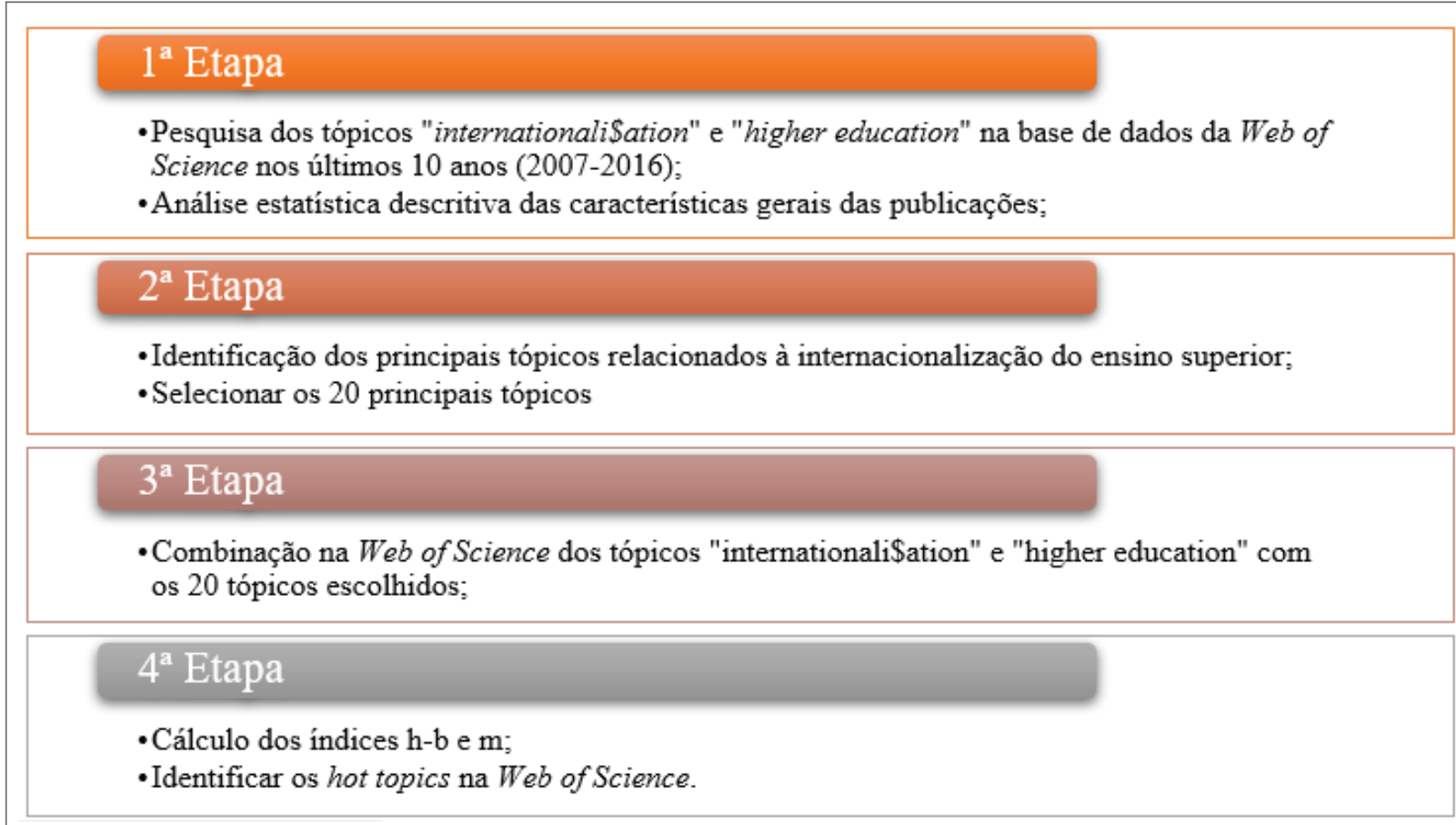

FIGURA 1 - Etapas do estudo

Fonte: elaborado pelos autores 
como os hot topics sobre a temática. Em linhas gerais, a amostra analisada apresentou uma média de 5,72 citações por publicação e um índice h-index de 37, o qual representa que 37 publicações foram citadas pelo menos por 37 vezes.

\section{Publicações por ano}

Analisando a produção científica dos estudos realizados sobre a internacionalização do ensino superior, no decorrer dos últimos 10 anos é possível notar, conforme visualizado no Gráfico I, um crescimento gradual no número de publicações, sendo que no período 2007-20I6 apresentou um crescimento médio de $36,36 \%$ ao ano, chegando a número de 252 publicações no ano de 2016, ano com o maior número de publicações. Desta maneira, é possível ratificar a importância que o tema tem recebido com o passar dos anos.

\section{Principais autores}

Referente à análise dos principais auto- res, o quadro 3 reflete os autores conforme o número de publicações, foram apontados todos os autores que possuíam 4 ou mais publicações no período analisado.

Dentre os autores informados, destacase o português Hugo Horta com II publicações no período analisado, constituindose como o autor com o maior número de publicações. Importante analisar que, mesmo sendo o autor com o maior número de publicações, a sua contribuição frente ao total de publicações representa aproximadamente $1 \%$ do total publicado, o que demonstra uma grande quantidade de autores com uma pequena quantidade de trabalhos publicados, essa informação é comprovada se considerarmos que a pesquisa retornou a existência de 2129 autores com pelo menos uma publicação no período analisado. Se considerarmos a soma das publicações no quadro 3, é possível verificar que os principais autores representam pouco menos de $10 \%$ do total de trabalhos publicados. Os

\section{GRÁFICO 1 - Número de publicações por ano pesquisado}

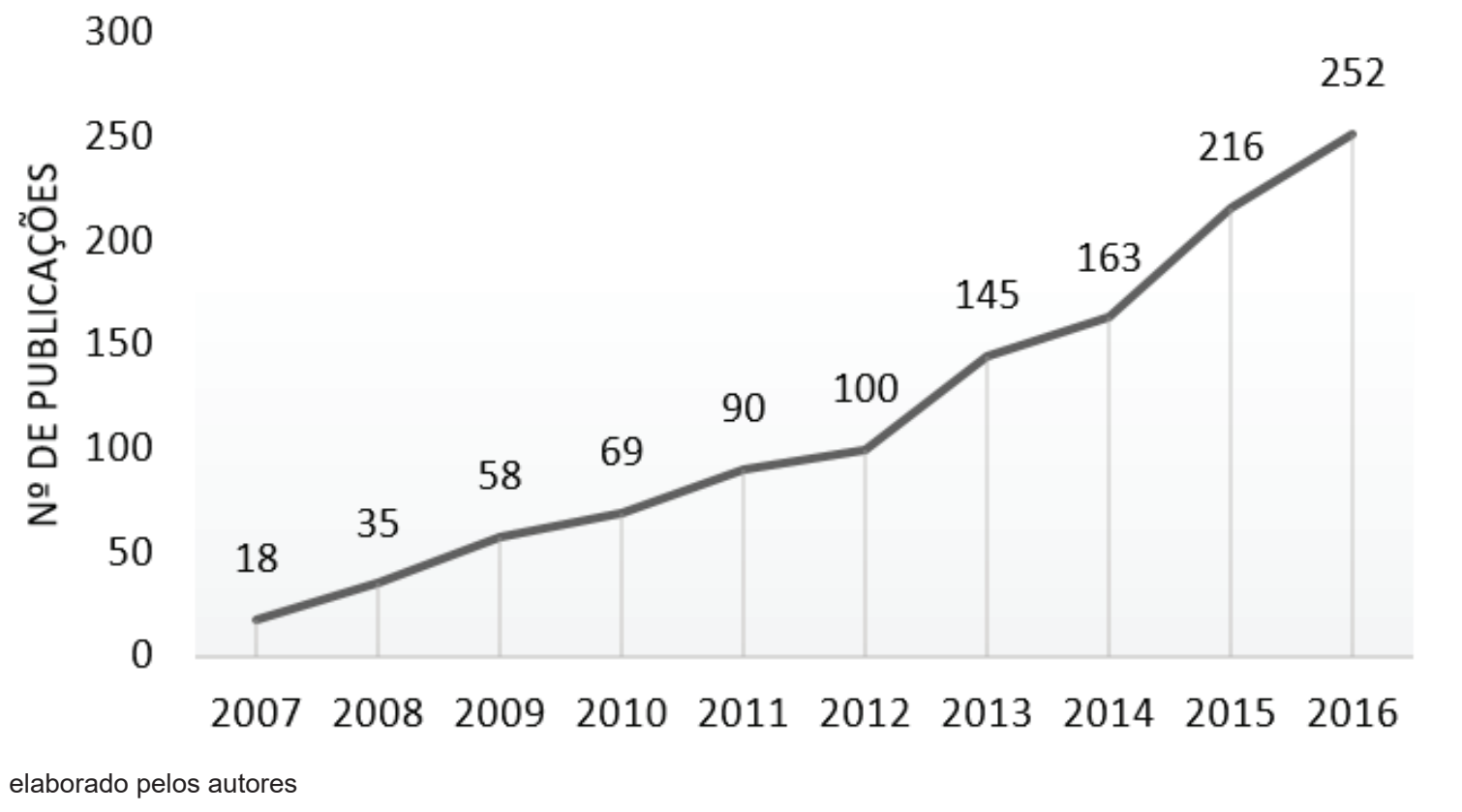


QUADRO 3 - Quantidade de artigos de acordo com o autor

\begin{tabular}{|c|c|c|}
\hline Autores & $N^{\circ}$ de Publicações & $\%$ de 1146 \\
\hline Horta, $\mathrm{H}$. & 11 & $0,960 \%$ \\
\hline Lasagabaster, D. & 6 & $0,524 \%$ \\
\hline Yemini, M. & 6 & $0,524 \%$ \\
\hline Moore, E. & 5 & $0,436 \%$ \\
\hline Wang, Y. & 5 & $0,436 \%$ \\
\hline Whitsed, C. & 5 & $0,436 \%$ \\
\hline Yonezawa, A. & 5 & $0,436 \%$ \\
\hline Chang, D. F. & 4 & $0,349 \%$ \\
\hline Cots, J. M. & 4 & $0,349 \%$ \\
\hline De Wit, H. & 4 & $0,349 \%$ \\
\hline Doiz, A. & 4 & $0,349 \%$ \\
\hline Green, W. & 4 & $0,349 \%$ \\
\hline Harrison, N. & 4 & $0,349 \%$ \\
\hline Heitor, M. & 4 & $0,349 \%$ \\
\hline Knight, J. & 4 & $0,349 \%$ \\
\hline Kondakci, Y. & 4 & $0,349 \%$ \\
\hline Llurda, E. & 4 & $0,349 \%$ \\
\hline Lo, W. Y. W. & 4 & $0,349 \%$ \\
\hline Oleksiyenko, A. & 4 & $0,349 \%$ \\
\hline Sidhu, R. & 4 & $0,349 \%$ \\
\hline Trahar, S. & 4 & $0,349 \%$ \\
\hline Volet, S. & 4 & $0,349 \%$ \\
\hline
\end{tabular}

Fonte: elaborado pelos autores

resultados encontrados são reforçados pelo estudo de Terra e Lengler (2016) que apontou que $91,2 \%$ dos autores pesquisados publicaram um único artigo no período entre 2000-2016.

Referente aos demais autores apresentados citam-se ainda os autores Lasagabaster, D. e Yemini, M., que possuem 6 publicações cada.

\section{Publicações por país}

O quadro 4 representa as publicações de acordo com país onde foi realizado, foram demonstrados no quadro 4 os 10 principais países no que concerne ao número de publicações dos tópicos analisados, insere-se na tabela ainda o Brasil que ocupa a II $^{\text {a }}$ posição em número de publicações. Verifica-se uma predominância dos estudos realizados na China, com um total de 197 artigos, seguido pela Inglaterra e Estados Unidos com 152 e 102 publicações, respectivamente. $\mathrm{O}$ Brasil encontra-se na $\mathrm{II}^{\mathrm{a}}$ posição com um total de 26 publicações ou $2,269 \%$ do total de estudos publicados.

Diferentemente do resultado encontrado nessa pesquisa, estudo realizado por Terra e Lengler (20l6) não verificou uma predominância dos estudos com origem na China, Estados Unidos e Austrália foram os países que mais se destacaram na pesquisa realizada por Terra e Lengler. A diferença entre os resultados pode ser explicada pelas diferentes bases de dados utilizadas. 
QUADRO 4 - Quantidade de publicações de acordo com o país

\begin{tabular}{|l|c|c|}
\hline País & $N^{\circ}$ de Publicações & $17,190 \%$ \\
\hline China & 197 & $13,264 \%$ \\
\hline Inglaterra & 152 & $9,162 \%$ \\
\hline Estados Unidos & 105 & $8,290 \%$ \\
\hline Austrália & 95 & $7,417 \%$ \\
\hline Espanha & 85 & $4,188 \%$ \\
\hline Canadá & 48 & $3,141 \%$ \\
\hline Romênia & 36 & $2,880 \%$ \\
\hline Rússia & 33 & $2,792 \%$ \\
\hline Alemanha & 32 & $2,356 \%$ \\
\hline Portugal & 27 & $2,269 \%$ \\
\hline Brasil & 26 & \\
\hline
\end{tabular}

Fonte: elaborado pelos autores

Referente a produção brasileira no período analisado, tem-se que de 2010 até 2014 , foi encontrada somente uma publicação anual, no ano de 2015 este número passou para 8, chegando a 14 publicações no ano de 2016. Não foram encontradas publicações nos anos anteriores a 2010 e no ano de 201 I. Com relação às organizações, nota-se uma grande dispersão neste quesito, existindo pelo menos 20 instituições com ao menos uma publicação, a Universidade Católica de Brasília, a Universidade Federal do Espírito Santo, a Universidade Federal do Rio Grande do Sul e a Universidade de São Paulo são as instituições que mais se destacaram com três publicações cada, juntas essas instituições representam 46, I52\% do que foi publicado no período.

\section{Publicações por tipo}

Oráfico 2 representa os tipos de documentos referente às publicações encontradas.

A maioria das publicações do período referem-se a artigos científicos, sendo que este tipo de documento representa aproximadamente $60 \%$ do total de publicações no pe- ríodo. Se considerarmos a soma dos artigos com os papers publicados, teremos quase a totalidade dos documentos publicados, sendo que a soma desses dois tipos de documentos representa $96,5 \%$ do total publicado.

Analisando-se a relação entre os tipos de documentos publicados com o país de origem da publicação julga-se importante destacar que as publicações chinesas, principal país em número de publicações, o artigo científico não é o principal tipo de documento publicado. Os artigos científicos representam pouco mais de $30 \%$ das publicações chinesas, enquanto os papers representam 69\%. Esta situação invertesse se considerarmos as publicações oriundas da Inglaterra, segunda colocada em número de publicações, onde os artigos representam $83 \%$ das publicações.

\section{Principais Instituições}

No quadro 5 estão representadas as 10 principais instituições que mais publicaram referente ao tema em estudo.

Ratificando a importância da China para os estudos de internacionalização do ensino superior, tem-se igualmente em primeiro lugar uma instituição chinesa com o 


\section{GRÁFICO 2 - Publicações de acordo com o tipo de documento}

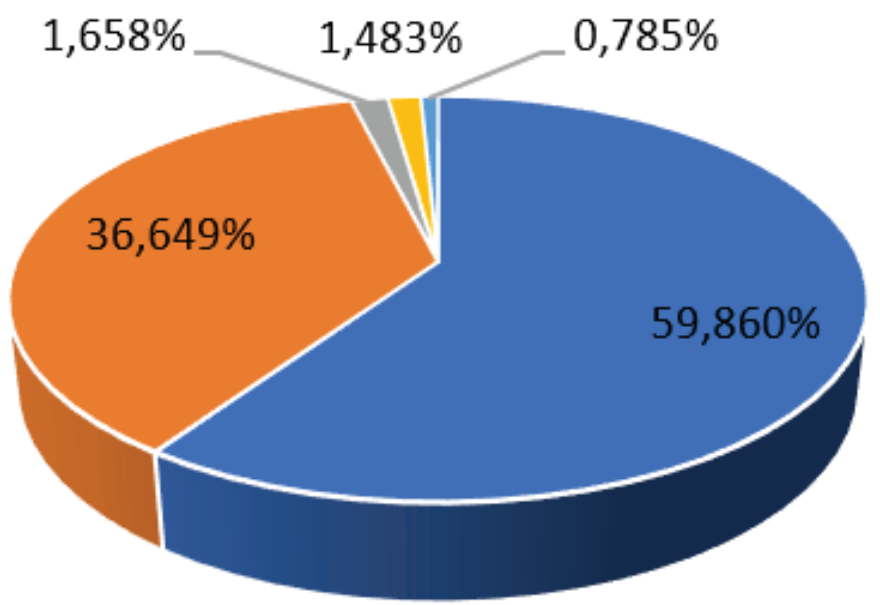

- Article

- Proceedings Paper

- Book Review

- Editorial Material

- Review

Fonte: elaborado pelos autores

QUADRO 5 - Número de publicações por instituição

\begin{tabular}{|l|c|c|}
\hline \multicolumn{1}{|c|}{ Instituição } & No de Publicações & $\%$ do total \\
\hline University of Hong Kong & 20 & $1,745 \%$ \\
\hline Murdoch University & 11 & $0,960 \%$ \\
\hline University of Nottingham & 11 & $0,960 \%$ \\
\hline University of British Columbia & 10 & $0,873 \%$ \\
\hline Universitat de Lleida & 10 & $0,873 \%$ \\
\hline Polytechinc University of Madrid & 10 & $0,873 \%$ \\
\hline Univ. Queensland & 10 & $0,873 \%$ \\
\hline Hong Kong Inst Educ & 9 & $0,785 \%$ \\
\hline Univ S Australia & 9 & $0,785 \%$ \\
\hline Univ Toronto & 9 & $0,785 \%$ \\
\hline
\end{tabular}

Fonte: elaborado pelos autores

maior número de publicações do período. A University of Hong Kong publicou 20 documentos durante o período informado.

A importância das publicações da University Hong Kong é reforçada pela média de citações apresentada e ainda pela soma total das citações recebidas no período. $A$ média de citações recebida por publicação foi de 16,85 citações por publicação, enquanto a soma total das citações foi de 337 citações para as 20 publicações analisadas, estes números são superiores as demais instituições analisadas. $O$ principal autor da instituição, considerando o número de publicações, é Hugo Horta, autor com o maior número de publicações no período analisado, o autor é mencionado em 5 publicações entre as 20 da instituição.

Ressalta-se ainda a importância das demais instituições para o desenvolvimento da temática, uma vez que as instituições citadas possuem pelo menos 9 publicações no período, o que representa aproximadamente uma publicação anual. É importante salientar que foram encontradas 940 instituições com pelo menos uma publicação no período. 


\section{Publicações por idioma}

No quadro 6 são representados os principais idiomas das publicações.

Fica evidente que quase a totalidade das publicações optam pela utilização do inglês para a linguagem dos seus trabalhos, dos I|46 resultados encontrados, aproximadamente $92 \%$ optaram pela utilização do inglês. Este resultado ratificado por DeWit (20l I) que trata o inglês como a linguagem da comunicação científica. Os idiomas chinês e espanhol, possuem, respectivamente, ○ $2^{\circ}$ e $3^{\circ}$ lugares como idioma das publicações, cada idioma apresentou 24 trabalhos.

Considerando-se os resultados encontrados, que ressaltam a utilização do inglês como a linguagem da comunicação científica, as publicações brasileiras demonstram certo contraponto aos resultados encontrados, uma vez que, das publicações nacionais analisadas, houve uma igualdade em número de publicações realizadas em português e inglês, este ponto pode demonstrar uma deficiência de autores nacionais em publicar em outros idiomas. Esta deficiência pode ser corroborada ao analisar as obras do autor com o maior número de publicações no período, Hugo Horta, autor português, onde as suas II publicações foram realizadas em inglês e ainda ao verificar que nos principais países analisados, as publicações em inglês são superiores aos demais idiomas.

\section{Publicações por área de pesquisa}

No quadro 7 são apresentadas as 10 principais áreas de pesquisa para os estudos em internacionalização do ensino superior.

A grande maioria dos estudos está vinculado à área de pesquisa voltada a pesquisa educacional, aproximadamente $67 \%$ dos estudos estão vinculados a essa área. Os estudos relacionados à área da economia e das ciências sociais também apresentaram números expressivos. Somados, os estudos dessas três áreas de pesquisa, representam aproximadamente $86 \%$ dos estudos realizados na temática de internacionalização do ensino superior.

\section{Fontes de publicação}

No quadro 8 são demonstradas as 10 principais fontes de publicações sobre a temática.

QUADRO 6 - Número de publicações de acordo com o idioma utilizado

\begin{tabular}{|l|c|c|}
\hline & No de Publicações & $\%$ de 1146 \\
\hline Inglês & 1057 & $92,234 \%$ \\
\hline Chinês & 24 & $2,094 \%$ \\
\hline Espanhol & 24 & $2,094 \%$ \\
\hline Alemão & 13 & $1,134 \%$ \\
\hline Português & 13 & $1,134 \%$ \\
\hline Russo & 9 & $0,785 \%$ \\
\hline Turco & 2 & $0,175 \%$ \\
\hline Africâner & 1 & $0,087 \%$ \\
\hline Holandês & 1 & $0,087 \%$ \\
\hline Letão & 1 & $0,087 \%$ \\
\hline Polonês & 1 & $0,087 \%$ \\
\hline
\end{tabular}

Fonte: elaborado pelos autores 
QUADRO 7 - Número de publicações conforme a área de pesquisa

\begin{tabular}{|l|c|c|}
\hline \multicolumn{1}{|c|}{ Área de Pesquisa } & $N^{\circ}$ de Publicações & $\%$ de 1146 \\
\hline Education Educational Research (Formação acadêmica pesquisa educacional) & 770 & $67,190 \%$ \\
\hline Business Economics (Economia) & 118 & $10,297 \%$ \\
\hline Social Sciences Other Topics (Ciências Sociais e outros tópicos) & 101 & $8,813 \%$ \\
\hline Linguistics (Linguística) & 55 & $4,799 \%$ \\
\hline Computer Science (Ciência da Computação) & 48 & $4,188 \%$ \\
\hline Engineering (Engenharia) & 34 & $2,967 \%$ \\
\hline Public Administration (Administração Pública) & 32 & $2,792 \%$ \\
\hline Geography (Geografia) & 21 & $1,832 \%$ \\
\hline Sociology (Sociologia) & 20 & $1,745 \%$ \\
\hline Psychology (Psicologia) & 19 & $1,658 \%$ \\
\hline
\end{tabular}

Fonte: elaborado pelos autores

QUADRO 8 - Número de publicações de acordo com a fonte de publicação

\begin{tabular}{|l|c|c|}
\hline \multicolumn{1}{|c|}{ Fonte de publicação } & No de Publicações & $\%$ de 1146 \\
\hline Journal of Studies in International Education & 104 & $9,075 \%$ \\
\hline Higher Education & 53 & $4,625 \%$ \\
\hline Edulearn Proceedings & 44 & $3,839 \%$ \\
\hline Procedia Social and Behavorial Sciences & 39 & $3,403 \%$ \\
\hline Inted Proceedings & 34 & $2,967 \%$ \\
\hline Studies in Higher Education & 22 & $1,920 \%$ \\
\hline Advances in Social Science Education and Humanities Research & 19 & $1,658 \%$ \\
\hline Advances in Education Research & 18 & $1,571 \%$ \\
\hline Compare a Journal of Comparative and International Education & 18 & $1,571 \%$ \\
\hline Higher Education Policy & 18 & $1,571 \%$ \\
\hline
\end{tabular}

Fonte: elaborado pelos autores

No que concerne às fontes de publicação percebe-se a existência de inúmeras fontes, sendo que entre as dez principais instituições, foram publicados no mínimo I 8 trabalhos. $O$ Journal of Studies in International Education é a fonte com o maior número de publicações, sendo 104 no total, seguido do Higher Education que possui quase metade do número de publicações da principal fonte encontrada, sendo 53 publicações no total.A importância do Journal of Studies in International Education para os estudos em internacionalização do ensino superior é corroborada pelos estudos de Terra e Lengler (2016) que apontou está fonte como responsável por 40,8\% das publicações analisadas.

\section{Hot topics}

Após uma análise prévia das publicações encontradas na base de dados da Web of Science foram determinados os principais tópicos relacionados à internacionalização do ensino superior. Foram encontrados 45 tópicos iniciais, dos quais foram selecionados os 20 principais de acordo com $n^{\circ}$ de publicações existentes na base de dados utilizada.

Posteriormente foi realizado o cruzamento de cada um dos 20 tópicos selecionados com os termos "internationali\$ation" e "higher education", salienta-se que foram utilizados os mesmos critérios da pesquisa inicial, pesquisa por tópicos, na base de dados principal da Web of Science e no período compreendido entre 2007- 
QUADRO 9 - Hot topics nos estudos sobre internacionalização do ensino superior

\begin{tabular}{|c|c|c|c|c|}
\hline Tópico & $\begin{array}{c}\mathrm{N}^{\circ} \text { de } \\
\text { Publicações }\end{array}$ & $\begin{array}{c}\mathrm{N}^{0} \text { de Publicações } \\
\text { do Cruzamento }\end{array}$ & $h-b$ & $\mathrm{~m}$ \\
\hline Care (cuidado) & 701.595 & 15 & 2 & 0,2 \\
\hline Resource (recurso) & 515.459 & 40 & 8 & 0,8 \\
\hline Student (estudante) & 343.994 & 303 & 20 & 2,0 \\
\hline China & 317.234 & 147 & 10 & 1,0 \\
\hline University (universidade) & 314.074 & 751 & 25 & 2,5 \\
\hline Diversity (diversidade) & 312.237 & 87 & 13 & 1,3 \\
\hline Identity (identidade) & 179.810 & 55 & 8 & 0,8 \\
\hline Teaching (ensino) & 176.568 & 312 & 16 & 1,6 \\
\hline Mobility (mobilidade) & 174.610 & 201 & 14 & 1,4 \\
\hline Ranking & 149.372 & 20 & 6 & 0,6 \\
\hline Responsibility (responsabilidade) & 89.424 & 26 & 4 & 0,4 \\
\hline Governance (governança) & 60.967 & 29 & 6 & 0,6 \\
\hline Career (carreira) & 43.956 & 24 & 7 & 0,7 \\
\hline Globali\$ation ${ }^{1}$ (globalização) & 33.005 & 298 & 21 & 2,1 \\
\hline Branding (marca) & 31.012 & 2 & 1 & 0,1 \\
\hline Socioeconomic status & 28.012 & 1 & 1 & 0,1 \\
\hline Hong Kong & 26.531 & 45 & 9 & 0,9 \\
\hline Singapore & 15.848 & 17 & 6 & 0,6 \\
\hline South Korea & 14.582 & 9 & 5 & 0,5 \\
\hline Middle East & 14.164 & 8 & 3 & 0,3 \\
\hline
\end{tabular}

Fonte: elaborado pelos autores

2016. A partir deste cruzamento foi possível determinar os índices h-b e m para cada tópico selecionado.

No quadro 9 é possível verificar os tópicos selecionados, o número de publicações para cada tópico, bem como o número de publicações encontradas no cruzamento entre os tópicos e ainda os índices h-b e m.

Seguindo-se as orientações propostas por Banks (2006) onde podem ser considerados hot topics ou tópicos quentes aqueles que apresentaram um índice $\mathrm{m}$ superior a 2. Desta maneira podem ser considerados hot topics os tópicos "universidade" e "globalização", que apresentaram índice m, respectivamente, de 2,5 e 2,I. Frisa-se, no entanto, que estes termos podem ser considerados correlatos aos termos "internacionalização" e "ensino superior", não representando especificamente uma área de estudo. De acordo com Terra e Lengler (2016) existe uma diversidade nos estudos em internacionalização que vão desde a definição de conceitos até as práticas em determinada região, o que leva a entender a ampla possibilidade de estudos na área de internacionalização do ensino superior.

De acordo com a classificação de Banks (2006) os termos resource (recurso), student (estudante), diversity (diversidade), identity (identidade), teaching (ensino), mobility (mobilidade), ranking, governance (go-

\footnotetext{
I Devido a existência do termo com diferentes grafias, foi utilizado o símbolo \$ para englobar o resultado com os diversos tipos de grafia
} 
vernança) e career (carreira) podem ser considerados tópicos emergentes. Dentre estes termos salienta-se a importância e o crescimento dos estudos relacionados a mobilidade de pessoas, tanto alunos quanto professores, como uma grande possibilidade de se tornar um hot topic.

Salienta-se ainda os tópicos China e Hong Kong que, em decorrência da quantidade de estudos realizados no país, podem ser considerados tópicos emergentes para os estudos em internacionalização do ensino superior.

Ademais, os tópicos que apresentaram $\mathrm{m}$ $\leq 0,5$ podem ser de interesse para pesquisadores em um campo específico de pesquisa.

\section{CONCLUSÃO}

O objetivo deste estudo foi explorar a produção acadêmica do tema internacionalização do ensino superior durante o período de 2007 a 2016 na base de dados da Web of Science, apresentando as características gerais das publicações, bem como identificar hot topics da temática estudada.

Foi possível notar um crescimento nas publicações no decorrer do período analisado, sendo que a maioria das publicações provem da China, Inglaterra e Estados Unidos e utilizam o inglês como linguagem predominante das publicações, sendo que o formato em artigo foi o tipo utilizado em mais da metade das publicações estudadas.

Encontrou-se ainda uma grande dispersão das publicações no que se refere aos autores, sendo que o principal autor (Hugo Horta) representa menos de $1 \%$ do total das publicações pesquisadas. $\bigcirc$ Journal of Studies in International Education foi encontrada como a principal fonte das publicações pesquisadas. A pesquisa educacional foi a área temática mais relevante para os resultados encontrados.
Os hot topics encontrados foram os tópicos "universidade" e "globalização", que podem ser considerados correlatos aos termos pesquisados e não uma área específica de estudo. Termos como "mobilidade" e "estudantes" podem ser considerados temas emergentes para os estudos em internacionalização do ensino superior.

Os dados apresentados permitiram verificar uma grande dispersão nos estudos sobre o tema, no entanto não foram encontrados grandes clusters de estudos, seja de autores ou instituições, ou uma área preponderante. É importante notar que o tema está em evolução no cenário mundial.

Salienta-se que este estudo não esgota a análise das publicações relacionadas ao tema, ao contrário, busca ampliar as discussões sobre o tema, jogar luz sobre a temática. Para tanto, outras análises, tanto qualitativas quanto quantitativas, devem ser realizadas objetivando um aprofundamento da análise.

A utilização de apenas uma base de dados foi um dos principais limitadores do estudo, entende-se que estudos futuros possam utilizar outras bases de dados internacionais e nacionais, ampliando e relacionando os resultados encontrados. Outro fator que pode ser explorado em estudos futuros é a combinação de outros termos que possibilitem ampliar o conhecimento sobre os estudos em internacionalização do ensino superior. Tem-se ainda a necessidade de desenvolver estudos que analisem a relação entre autores, instituições e periódicos, identificado redes de relacionamento, clusters e as principais cocitações existentes. Ao desenvolver este grupo de estudos será possível estabelecer uma visão geral dos estudos que estão sendo desenvolvidos na área e aprofundar uma análise da área. 


\section{REFERÊNCIAS}

ALTBACH, P. G.; KNIGHT, J. The internationalization of higher education: Motivations and realities. Journal of studies in international education, $v$. II, n. 3-4, p. 290-305, 2007.

ALTBACH, P. Globalization and the University: Realities in an Unequal World. In: ALTBACH, P.; FOREST, J. (eds.). International Handbook of Higher Education. Dordrecht: Springer, 2006.

ARAUJO, C. A. Bibliometria: evolução histórica e questões atuais. Em Questão: Revista da Faculdade de Biblioteconomia e Comunicação da UFRGS, v. I2, n. I, p. II-32, jan./jun. 2006. Disponível em: <http://revistas. univerciencia.org/index.php/revistaemquestao/article/viewFile/3707/3495>. Acesso em: II nov. 2017.

BANKS, M. G. An extension of the Hirsch index: indexing scientific topics and compounds. 2006. Disponível em: <http://www.arxiv. org/abs/physics/0604216>. Acesso em: 12 nov. 2017.

BORGES, G. D. R.; AMAL, M. Internacionalização de cursos stricto sensu: uma investigação sobre a distância psíquica e as práticas adotadas. Revista Gestão Universitária na América LatinaGUAL, v. 9, n. 2, p. 260-28I, 2016. COORDENAÇÃO DEAPERFEIÇOAMENTO DE PESSOAL DE NÍVEL

SUPERIOR. Plano Nacional de Pós-Graduação - PNG 201 I/2020 Vol. I - Brasilia, DF: CAPES, 2010.

COORDENAÇÃO DEAPERFEIÇOAMENTO DE PESSOAL DE NÍVEL

SUPERIOR. Acervo. 2017. Disponível em: <https://www.periodicos. capes.gov.br/?option $=$ com_pcollection \&mn $=70 \& s m n=79 \&$ -
cid=8I>.Acesso em: 01 out. 2017. DAL-SOTO, F;:ALVES, J. N.;SOUZA,Y. S. de. A produção científica sobre internacionalização da educação superior na Web of Science: características gerais e metodológicas. Educação em Revista, v. 32, n. 4, p. 229-249, 2016.

DE WIT, H. Globalisation and internationalisation of higher education. Revista de Universidad y Sociedad del Conocimiento (RUSC), v. 8, n. 2, p. 24l-247, 2011.

DUTRA, I. I. C.; MARANHÃO, R. K. D. A. Internacionalização do ensino superior: um estudo sobre barreiras e possibilidades. Administração: Ensino e Pesquisa, v. 18, n. I, p. 9-38, 2017.

FIGUEIRÓ, P. S.; RAUFFLET, E. Sustainability in higher education: a systematic review with focus on management education. Journal of Cleaner Production, v. 106, p. 22-33, 2015.

HIRSCH, J. E. An index to quantify an individual's scientific research output. Proceedings of the National Academy of Sciences of the United States of America, v. 102, n. 46, p. 16.569 16.572, 2005.

JACKSON, J. Globalization, internationalization, and short-term stays abroad. International Journal of Intercultural Relations, v. 32, n. 4, p. 349-358, 2008.

$\mathrm{KNIGHT}$, J. Higher Education in Turmoil:The Changing World of Internationalization. Rotterdam: Sense Publishers, 2008.

LIMA, M. C., CONTEL, F. B. Internacionalização da educação superior: Nações ativas, nações passivas e a geopolítica do conhecimento. São Paulo: Alameda, 2011 .
MACEDO, M. A. S.; CASA NOVA, S. P. C.;ALMEIDA, K. Mapeamento e análise bibliométrica da utilização da análise envoltória de dados (DEA) em estudos de contabilidade e administração. CGG - Contabilidade, Gestão e Governança, v.I2, n.3, p.87-I0I, set./ dez. 2009.

MARINGE, F., FOSKETT, N. Globalization and Internationalization in Higher Education: Theoretical, Strategic and Management Perspectives. London: Continuum International Publishing Group. 2010.

MARRARA, T. Internacionalização da Pós-Graduação: objetivos, formas e avaliação. Revista Brasileira de Pós-Graduação, v. 4, n. 8, p. 245-262, 2007.

MOROSINI, M. C.; FERNANDES, C. M. B.; LEITE, D. et al. A qualidade da educação superior e o complexo exercício de propor indicadores. Revista Brasileira de Educação, v. 2 I, n. 64, p. I3-37, 2016.

$\mathrm{RAASCH}, \mathrm{C}$. et al. The rise and fall of interdisciplinary research: the case of open source innovation. Research Policy, v. 42, n. 5, p. II38-II5I, 2013.

SCOTT, P. The Global Dimension: Internationalising Higher Education. In: DE WIT, B. K. H. (ed.). Internationalization in Higher Education: European Responses to the Global Perspective. Amsterdam: European Association for International Education and the European Higher Education Society, 2005.

STALLIVIERI, L. O processo de internacionalização nas instituições de ensino superior. Educação Brasileira: Revista do Conselho de Reitores das Universidades Brasileiras, Brasília, v. 24, n. 
48, p. 35-57, 2002.

TEICHLER, U. The Changing Debate on Internationalisation of Higher Education. Higher Education, v.
48, n. I, p. 5-26, 2004.

TERRA, A., LENGLER, J. F. B. internacionalização do ensino superior: Para onde estamos indo? Uma análise das publicações a partir da virada do milênio. Revista do CEPE. Santa Cruz do Sul, n. 44, p. I47-I68, jul./dez. 2016. 